\title{
On a Codimension Three Bifurcation Arising in a Simple Dynamo Model
}

\author{
Anne C. Skeldon ${ }^{\mathrm{a}, 1}$ and Irene M. Moroz ${ }^{\mathrm{b}}$ \\ ${ }^{a}$ Department of Mathematics, City University, Northampton Square, London \\ EC1V 0HB, England \\ b Mathematical Institute, 24-29 St Giles', Oxford OX1 3LB, England
}

\begin{abstract}
In this paper we investigate the dynamics associated with a degenerate codimension two Takens-Bogdanov bifurcation which arises in a recently derived model for selfexciting dynamo action introduced by Hide et al. [1]. The general unfolding of such a codimension three bifurcation has already been discussed in an abstract setting by $\mathrm{Li}$ and Rousseau [4]. Here we describe the unfolding scenario in the context of the dynamo problem. In particular we compare the behaviour predicted by the normal form analysis with a bifurcation study of the full dynamo equations in the neighbourhood of the codimension three point.
\end{abstract}

\section{Introduction}

In a recent paper, Hide et al. [1] proposed a model for self-exciting dynamo action in which a Faraday disk and coil are arranged in series with either a capacitor or a motor. The non-dimensional system of nonlinear ordinary differential equations, governing such a dynamo are

$$
\begin{aligned}
& \dot{x}=x(y-1)-\beta z, \\
& \dot{y}=\alpha\left(1-x^{2}\right)-\kappa y, \\
& \dot{z}=x-\lambda z,
\end{aligned}
$$

\footnotetext{
1 For correspondance
} 
where $\dot{x}=\frac{d x}{d t}$, etc. Here $x(t)$ is the rescaled electric current in the system, $y(t)$ denotes the angular rotation rate of the disk and $\mathrm{z}(\mathrm{t})$ measures either the charge of the capacitor or the angular speed of rotation of the motor. Four dimensionless parameters control the dynamics of the system. In (1), $\alpha$ measures the applied couple and $\kappa$ the mechanical friction. For the motor, $\beta^{-1}$ measures the moment of inertia of the armature and $\lambda$ the mechanical friction in the motor, while for the capacitor $\beta^{-1}$ and $\lambda$ measure the capacitance and leakage resistance respectively. The reader is referred to [1] for further details.

A local analysis about the equilibrium state,

$$
\left(x_{0}, y_{0}, z_{0}\right)=(0, \alpha / \kappa, 0)
$$

revealed the existence of a codimension two double-zero bifurcation as the organising centre. The associated normal form has a degeneracy when $\lambda=\frac{3}{2} \kappa$. The objective of this paper is to extend the normal form calculations of [1] to quintic order in order to study the unfolding of this codimension three bifurcation in the context of the dynamo model. (Because of the invariance of equations (1) under the transformation $(x, y, z) \rightarrow(-x, y,-z)$, the normal form has reflectional symmetry and so contains only odd powers of the dependent variables.) The resulting normal form has already been studied in an abstract context by Li and Rousseau [4], who provided general bifurcation sets for the two qualitatively different scenarios which can occur. In this paper, we reconcile the bifurcation set relevant to the dynamo problem with its counterpart in the physical space of the full dynamo equations (1), using the numerical bifurcation package AUTO.

There have been other studies of codimension three bifurcations in the literature (see for example Dangelmayr et al. [2] and Moroz [3]), but these studies either discuss a different degeneracy to the one considered here [2], or merely derive the quintic order normal form and make no attempt at explaining the 
behaviour close to the degeneracy [3]. We shall return to this later.

The paper is organised as follows. In $\S 2$ we summarise the normal form calculations that lead to the quintic order normal form, giving general analytical expressions for the various coefficients. $\S 3$ contains a brief discussion of the unfolding analysis of [4], placed in the context of the dynamo model. In $\S 4$ we compare the numerically computed bifurcation set of the dynamo equations (1) with the behaviour predicted by the unfolding scenario for appropriate choices of the control parameters. We draw our conclusions in $\S 5$, suggesting future directions of the work.

\section{The Normal Form Calculations}

The calculation of the normal form can be simplified if we translate the equilibrium state $\left(x_{0}, y_{0}, z_{0}\right)=(0, \alpha / \kappa, 0)$ to the origin by introducing the new variable

$$
Y=y-\alpha / \kappa
$$

Then equations (1) becomes

$$
\begin{aligned}
\dot{x} & =(\bar{\alpha}-1) x-\beta z+x Y, \\
\dot{Y} & =-\kappa\left(Y+\bar{\alpha} x^{2}\right), \\
\dot{z} & =x-\lambda z
\end{aligned}
$$

where $\bar{\alpha}=\alpha / \kappa$. A linear stability analysis of (4) about the trivial equilibrium state shows that there is a line of steady bifurcations given by

$$
\bar{\alpha}=\beta / \lambda+1
$$

and a line of Hopf bifurcations along

$$
\bar{\alpha}=\lambda+1
$$


provided $\beta \geq \lambda^{2}$. The Hopf curve branches off the steady curve when

$$
(\bar{\alpha}, \beta)=\left(\lambda+1, \lambda^{2}\right)
$$

At this point the frequency of the oscillations is zero and we have a codimension two double-zero bifurcation. The reader is referred to [1] for details.

Using standard centre manifold reduction techniques it can be shown that the dynamics in the neighbourhood of the codimension two point (7) are given by the equations

$$
\begin{aligned}
& \dot{\eta}=\xi, \\
& \dot{\xi}=\epsilon_{1} \eta+\epsilon_{2} \xi+a_{1} \eta^{3}+b_{1} \xi \eta^{2}++c \xi^{2} \eta+d \xi^{3},
\end{aligned}
$$

where

$$
\xi=x-\lambda z, \quad \eta=x
$$

and

$$
\begin{aligned}
\epsilon_{1}=\lambda \tilde{\alpha}-\tilde{\beta}, & \epsilon_{2}=\tilde{\alpha}, \\
a_{1}=-\lambda^{3}(1+\lambda), & b_{1}=\lambda^{2}(1+\lambda)\left(2 \frac{\lambda}{\kappa}-3\right), \\
c=-\lambda(1+\lambda)\left(2 \frac{\lambda^{2}}{\kappa^{2}}-4 \frac{\lambda}{\kappa}+3\right), & d=-(1+\lambda)\left(2 \frac{\lambda^{2}}{\kappa^{2}}-2 \frac{\lambda}{\kappa}+1\right) .
\end{aligned}
$$

Here, $\tilde{\alpha}, \tilde{\beta}$ denote small perturbations away from the codimension two point (7). On using the further simplification of normal forms (for example see $\S 7.3$ of Guckenheimer and Holmes [5]), near identity transformations enable two of the cubic coefficients to be removed leaving,

$$
\begin{aligned}
& \dot{u}=v, \\
& \dot{v}=\epsilon_{1} u+\epsilon_{2} v+a_{1} u^{3}+b_{1} u^{2} v
\end{aligned}
$$


where $(u, v)=(\eta, \xi)+$ higher order terms. The codimension two point now occurs when $\epsilon_{1}=\epsilon_{2}=0$. Two points should be noted at this stage: firstly, the coefficients in equation (8) in general depend on $\epsilon_{i}$ and we have assumed that we are sufficiently close to the codimension two point that this dependence may be neglected; secondly, the coefficients $a_{1}$ and $b_{1}$ are unchanged in the normal form transformations. These two points become significant when we try to relate results from the full equations to the predictions of the normal form and we will return to them in $\S 4$ below.

From (11) we see that $b_{1}$ can pass through zero when $\lambda=\frac{3}{2} \kappa$. When this happens, the cubic normal form (11) is unable to describe the behaviour near the codimension two point and we need to include higher order terms in both the centre manifold reduction and in the normal form equations. The reflectional symmetry satisfied by equations (1) and (4) mean that only odd powers of $\mathrm{u}$ and $\mathrm{v}$ enter into the normal form and we therefore obtain:

$$
\begin{aligned}
& \dot{u}=v \\
& \dot{v}=\epsilon_{1} u+\epsilon_{2} v+a_{1} u^{3}+b_{1} u^{2} v+a_{2} u^{5}+b_{2} u^{4} v
\end{aligned}
$$

where the coefficients of the quintic terms in (12) are

$$
a_{2}=-\frac{45}{2} \lambda^{3}(1+\lambda)^{2}, \quad b_{2}=\frac{3}{2} \lambda^{4}(1+\lambda)^{2}
$$

The coefficient $b_{1}$ takes on the role of a third bifurcation parameter and we have the codimension three bifurcation problem studied by Li and Rousseau [4]. For the physically realistic case of $\lambda>0, a_{1}$ and $b_{2}$ are always negative, $a_{2}$ is always positive, while $\epsilon_{1}, \epsilon_{2}$ and $b_{1}$ can take either sign. We shall discuss the unfolding of (12) in the next section.

Dangelmayr et al. [2] investigated the codimension three bifurcation which arises in the problem of a laser with saturable absorber when the coefficient ' $a_{1}$ ' in (8) vanishes. They only consider the quintic term ' $u$ ' as being relevant 
to the unfolding of this degeneracy. Here we require both ' $u^{5}$ ' and ' $u{ }^{4} v$ ' terms. In a study of cubic order degeneracies that can arise in a model for twodimensional Langmuir circulations, Moroz [3] derived a quintic order normal form like (9) when either the coefficient corresponding to ' $a_{1}$ ' or ' $b_{1}$ ' in that problem vanished. However no attempt was made to unfold the bifurcation.

\section{The Unfolding Scenario}

Li and Rousseau [4] were able to simplify the quintic normal form still further by introducing a nonlinear change of variables which has the effect of eliminating the $u^{5}$ term in (12). This enables (12) to be re-scaled and re-expressed as a perturbed Hamiltonian system in which the Hamiltonian part is the same as for the cubic normal form (11), while all the remaining non-Hamiltonian terms are of the same order of magnitude. For completeness we briefly summarise their argument here.

Setting $\epsilon_{1}=\epsilon_{2}=0$, we can re-write (12) as

$$
v d v-\left(a_{1} u^{3}+a_{2} u^{5}\right) d u-v\left(b_{1} u^{2}+b_{2} u^{4}\right) d u=0
$$

Following [4], we introduce a new variable U defined by

$$
\frac{a_{1} U^{4}}{4}=\frac{a_{1} u^{4}}{4}+\frac{a_{2} u^{6}}{6}
$$

On differentiating both sides of (15) and substituting into (14) we get

$$
v d v-a_{1} U^{3} d U-v\left(b_{1} u^{2}+b_{2} u^{4}\right) d u=0
$$

Next we express $u$ and $d u$ in terms of $U$ and $d U$ to obtain

$$
v d v-a_{1} U^{3} d U-v\left(c_{1} U^{2}+c_{2} U^{4}\right) d U=0,
$$


which is equivalent to the normal form

$$
\begin{aligned}
\dot{U} & =v, \\
\dot{v} & =a_{1} U^{3}+c_{1} U^{2} v+c_{2} U^{4} v,
\end{aligned}
$$

where

$$
c_{1}=b_{1}, c_{2}=b_{2}-\frac{5 b_{1} a_{2}}{6 a_{1}}
$$

Finally we re-introduce the unfolding parameters to recover the simplified normal form

$$
\begin{aligned}
\dot{U} & =v \\
\dot{v} & =\epsilon_{1} U+\epsilon_{2} v+\epsilon_{3} U^{2} v+a_{1} U^{3}+c_{2} U^{4} v
\end{aligned}
$$

where we have denoted small departures from $c_{1}=0$ by $\epsilon_{3}$. Li and Rousseau [4] rescale (20) and consider the two cases:

$$
c_{2}=-1, a_{1}= \pm 1
$$

It is the $a_{1}=-1$ case that is relevant to our particular problem.

With the further change of coordinates

$$
U=\delta r, v=\delta^{2} s, t=\delta^{-1} \tau, \epsilon_{1}=-a_{1} \delta^{2} \mu_{0}, \epsilon_{2}=\delta^{4} \mu_{1}, \epsilon_{3}=\delta^{2} \mu_{2}
$$

(20) becomes the perturbed Hamiltonian system (see equation (3.2) of [4])

$$
\begin{aligned}
& r^{\prime}=s, \\
& s^{\prime}=-a_{1} \mu_{0} r+a_{1} r^{3}+\delta^{3}\left(\mu_{1}+\mu_{2} r^{2}-r^{4}\right) s,
\end{aligned}
$$

with Hamiltonian

$$
H=\frac{s^{2}}{2}+\frac{a_{1} \mu_{0} r^{2}}{2}-\frac{a_{1} r^{4}}{4}
$$


When $\delta=0$, (23) has three equilibria for $\mu_{0}>0$ and one equilibrium for $\mu_{0}<0$. The trivial state $(r, s)=(0,0)$ is a centre for $a_{1}=+1$ and a saddle point for $a_{1}=-1$; the two "finite-amplitude" states $(r, s)=\left( \pm \sqrt{\mu_{0}}, 0\right)$ are saddles for $a_{1}=+1$ and centres for $a_{1}=-1$, provided $\mu_{0}>0$.

Because of the removal of the $u^{5}$ term from the normal form, the Melnikov method (see, for example, Guckenheimer and Holmes [5]) can be applied directly to (23) in order to determine the location of global homoclinic and/or heteroclinic bifurcations in terms of the unfolding parameters. The paper by Li and Rousseau [4], supplemented by results proved in Chow et al [6] provides a comprehensive survey of the properties of the Melnikov integral

$$
M(h)=\int_{H=h}\left(\mu_{1} s+\mu_{2} r^{2} s-r^{4} s\right) d r
$$

and its zeros. The analytical treatment provides information on the maximum number of coexisting limit cycles that can surround each equilibrium state (termed internal limit cycles) and the number of large amplitude limit cycles surrounding all three equilibria (termed external limit cycles). Also discussed is the type and location of homclinic and heteroclinic orbits and the way in which these global bifurcations cause the destruction of the various limit cycles. The study culminates in the construction of schematic gyratory bifurcation diagrams for $a_{1}=-1$ and for $a_{1}=+1$.

In the next section we corroborate the unfolding scenario predicted by Li and Rousseau for $a_{1}=-1$, which is the case relevant to the dynamo problem, by using the bifurcation and path-following package AUTO. We then compare this with the computed bifurcation structure of the full dynamo equations. 


\section{Numerical Results}

\subsection{Computed bifurcation set for the normal form}

Li and Rousseau [4] presented their original results in the form of a bifurcation set on the half-sphere $\epsilon_{1} \geq 0$. In order to aid comparison with numerical results we have re-drawn their qualitative bifurcation set for the case $a_{1}=-1$ in a cube in $\left(\epsilon_{1}, \epsilon_{2}, \epsilon_{3}\right)$-parameter space, as shown in figure 1 . In this figure, the codimension three point occurs at the orgin at the intersection of five different bifurcation surfaces. The surface $\epsilon_{2}=0, \epsilon_{1} \leq 0$ is the surface of Hopf bifurcation points for which equation (6) holds. The plane $\epsilon_{1}=0$ is the plane of pitchfork bifurcations corresponding to equation (5). These two planes meet along the $\epsilon_{3}$ axis, and it is local to this axis for $\epsilon_{3} \neq 0$ that the codimension two analysis discussed in Hide et al. [1] is relevant. The three other surfaces shown correspond to surfaces of a second Hopf bifurcation, homoclinic bifurcations and saddle-node bifurcation of limit cycles.

In order to confirm the qualitative nature of the bifurcation set in figure 1 we have computed three planes from the normal form (20). These three surfaces, namely, $\epsilon_{3}=-0.1, \epsilon_{3}=0.1$ and $\epsilon_{2}=0.1$, are presented in figure 2 and correspond to the back, front and top of the cube shown in figure 1 respectively. In all three cases, qualitative phase plane portraits are included indicating the steady-state solutions (shown with crosses) and the limit cycles for each region: stable limit cycles are shown with a solid curve and unstable limit cycles with a dashed curve. Local to the origin in both figures 2(a) and 2(b) the bifurcation set is consistent with the codimension two analysis for $\epsilon_{3} \neq 0$. Indeed, figure $2(\mathrm{a})$, showing the case $\epsilon_{3}<0$, can be compared directly with the codimension two unfolding shown in figure 7.3.9 in Guckenheimer and Holmes [5].

In figure 2(a), starting in the third quadrant and travelling clockwise around 
the codimension two point at the origin, the following sequence of transitions occurs. Firstly, there is a single stable steady-state solution corresponding to the trivial solution. On traversing the $\operatorname{Hopf}_{1}$ bifurcation line at $\epsilon_{2}=0$, this trivial solution becomes unstable and a stable limit cycle is born. Continuing clockwise round the origin, at $\epsilon_{1}=0$ two further steady-state solutions are created at the pitchfork bifurcation. These steady-state solutions in turn bifurcate at the $\mathrm{Hopf}_{2}$ bifurcation line producing two, small amplitude, unstable limit cycles. These two unstable limit cycles grow until they become homoclinic to the trivial steady-state solution, and are replaced by a single large unstable limit cycle encircling all three steady-state solutions. The two large amplitude limit cycles, the smaller one of which was produced by the homoclinic bifurcation and is unstable, and the larger one of which was produced in the Hopf 1 bifurcation and is stable, then annihilate one another at the line of limit point bifurcations. This leaves just the three steady-state solutions. In turn, these are reduced to one steady-state solution at the pitchfork bifurcation line, and the phase portrait in the third quadrant is recovered.

Guckenheimer and Holmes note that the case $\epsilon_{3}>0$ can be obtained from that for $\epsilon_{3}<0$ by rescaling, specifically by mapping $\epsilon_{2} \mapsto-\epsilon_{2}, v \mapsto-v$ and reversing time. This correspondance between the two cases can be seen if figure 2 (a) is compared with figure 2(b) local to the origin. Indeed, starting now from the second quadrant and passing anti-clockwise around the origin the same sequence of bifurcations described for figure 2(a) occurs. The key difference

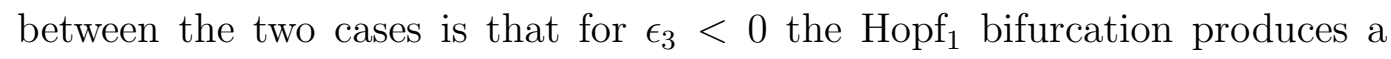
stable periodic orbit and the Hopf $f_{2}$ bifurcation produces unstable periodic orbits. However, for $\epsilon_{3}>0$ it is the $\mathrm{Hopf}_{2}$ bifurcation which produces stable periodic orbits and the $\mathrm{Hopf}_{1}$ bifurcation produces an unstable periodic orbit.

Note that in figure 2(b), for all the phase portraits for regions close to the origin, there is an additional large limit cycle. This is a feature which emerges 
from the codimension three analysis; it is not local to the origin for $\epsilon_{3} \neq 0$ and therefore, sufficiently close to the codimension two point, plays no role in the dynamics. However, this large amplitude limit cycle is instrumental in enabling the change in the stability of the periodic orbits created in the Hopf $_{1}$ bifurcation to occur as $\epsilon_{3}$ changes sign. Away from the origin, the effect of this large orbit can be seen in the cusp and resultant bending back of the limit point bifurcation line. This has the effect of removing the unstable

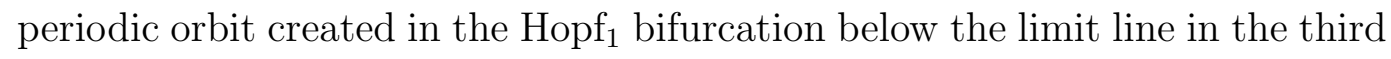
and fourth quadrants. In the third quadrant this means that for $\epsilon_{2}$ less than approximately -0.0012 , instead of one unstable periodic orbit, as predicted by the codimension two analysis, there are no periodic orbits. In the fourth quadrant, and extending into the first, the removal of the periodic orbit created

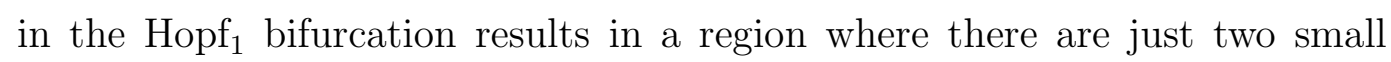
amplitude stable periodic orbits and no other limit cycles.

In figure 2(c) we show how the codimension three analysis enables the transition from stable to unstable periodic orbits at the $\mathrm{Hopf}_{2}$ bifurcation to take place. The bifurcation lines shown are all very close together: the axes have been rotated by $60^{\circ}$ to aid their visualisation. Consider taking an anticlockwise path, close to the edge of the figure, starting at the upper left. In the first region there are three steady-state solutions. These are supplemented by two large amplitude periodic orbits, the inner one unstable and the outer one stable, as the limit bifurcation line is crossed. The inner periodic orbit becomes homoclinic to the central steady-state solution, at the homoclinic line, and is subsequently replaced by two small amplitude, unstable, periodic orbits. These small periodic orbits then disappear along the $\mathrm{Hopf}_{2}$ bifurcation line, leaving just one large amplitude, stable, periodic orbit. This takes us to the far left of the figure. Now passing back to the right of the figure, but from a point towards the bottom, first a limit point bifurcation line is crossed. This creates two pairs of periodic orbits, one pair around each of the asymmet- 
ric steady-state solutions. Of each pair, the inner periodic orbit is stable and the outer, unstable. On crossing the homoclinic line, the two unstable orbits glue together and are replaced by one large unstable orbit. This is then destroyed, along with the large outer stable periodic orbit, at the second limit point bifurcation line, leaving just the two small amplitude, stable, limit cycles which surround the asymmetric steady-state solutions. These limit cycles then undergo $\mathrm{Hopf}_{2}$ bifurcations to return us to the original three steady-state solutions on the far right of the figure. Note that at the top of the figure, the small limit cycles surrounding the asymmetric steady-state solutions are unstable, as required for $\epsilon_{3}<0$, but at the bottom, they are stable, as required for $\epsilon_{3}>0$.

\subsection{Computed bifurcation set for the full equations}

We now turn to computations of the bifurcation set of the full equations (1). We computed bifurcation curves corresponding qualitatively to the front and back faces of the cube shown in figure 1 , that is planes such that $\epsilon_{3}=$ constant . In terms of the original variables,

$$
\epsilon_{3}=2 \lambda^{2}(1+\lambda)\left(\frac{\lambda}{\kappa}-\frac{3}{2}\right)
$$

Therefore, planes of constant $\epsilon_{3}$ may be obtained by fixing $\lambda$ and $\kappa$. As in section 4.1, we chose to compute the planes $\epsilon_{3}= \pm 0.1$ and fixed $\lambda=0.2$. This gave corresponding values for $\kappa$ of 0.07868 for $\epsilon_{3}=0.1$ and of 0.43636 for $\epsilon_{3}=-0.1$. The computed bifurcation set is shown in figure 3(a) for $\epsilon_{3}=-0.1$. The codimension two point occurs at $\alpha=0.523632, \beta=0.04$; the pitchfork bifurcation line occurs at $\alpha=2.1818 \beta+0.43636$ and the Hopf bifurcation line occurs at $\alpha=0.523632, \beta>0.04$. In the normal form these two bifurcation

lines correspond to $\epsilon_{1}=0$ and $\epsilon_{2}=0, \epsilon_{1}<0$ respectively. In order to aid comparison with figure 2 , we have plotted $\alpha$ against $\beta$, with $\beta$ increasing to 
the left and it is then readily seen that there is good qualitative agreement between figure 3(a) and figure 2(a).

The analogous diagram for $\epsilon_{3}=0.1$ is presented in figure 3(b). Local to the codimension two point at $\alpha=0.094416, \beta=0.04$, as shown by the expanded part of the diagram, there is good qualitative agreement with the bifurcation set shown in figure 2(b). The path of limit points was at first hard to find: for most parameter values our initial computations found the Hopf $\mathrm{f}_{1}$ bifurcation was supercritical. In the case presented in figure 3(b), for example, the Hopf $_{1}$ bifurcation is supercritical for $\beta>0.0416$ and is only subcritical for $0.04<\beta<0.0416$. This is in contrast to the normal form for which the Hopf bifurcation is subcritical whenever $\epsilon_{3}>0$. In fact, the position of the degenerate Hopf bifurcation in the $(\alpha, \beta, \kappa, \lambda)$-space, which marks the transition between sub- and supercritical Hopf bifurcation, cannot be reconstructed from knowledge of the normal form, even local to the codimension three point. The reasons for this are twofold; firstly because of the neglect of the parameter dependence of the cubic coefficients in the derivation of the centre manifold equations, secondly, and more importantly, because information on the position of the Hopf degeneracy is lost in the normal form transformations. In fact, the position of the degeneracy is determined by the cubic coefficients $a_{1}$ and $c$ of the centre manifold equations (8). In converting the centre manifold equations to the normal form, $a_{1}$ is left unchanged while $c$ is removed, thus moving the position of the degeneracy. While the first of these problems can be remedied, the second cannot, and no more than qualitative agreement can be expected between the normal form and the full equations.

In order to resolve this difficulty and enable us to find the region where the Hopf $_{1}$ bifurcation is subcritical, we return to the full equations and perform a centre manifold reduction and corresponding normal form transformations

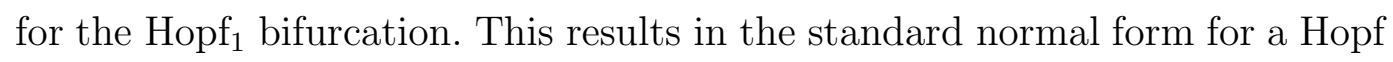


bifurcation with a cubic coefficient which is zero when

$$
\tilde{\beta}=\frac{\kappa^{2}}{4}\left(\frac{\lambda}{\kappa}-\frac{3}{2}\right),
$$

where $\tilde{\beta}=\beta-\lambda^{2}$ measures the distance from the codimension two point. In terms of the unfolding parameters $\epsilon_{i}$ this gives

$$
\epsilon_{1}=-\frac{1}{8} \frac{\kappa^{2}}{\lambda^{4}} \frac{\epsilon_{3}}{(1+\lambda)}
$$

Hence, although the Hopf bifurcation is subcritical for $\epsilon_{3}>0$ sufficiently close to the codimension three point, this region of subcriticality is quite small: our choice of $\lambda=0.2$ was chosen to approximately maximise the subcritical region.

As well as the AUTO calculations described above, we have independently performed numerical integrations of the coupled dynamo equations (1) for typical parameter values that are appropriate to figures 3(a) and 3(b). Our numerical integrations were able to verify the predictions of both the bifurcation analysis and AUTO computations.

\section{Conclusions}

In this paper we have carried out a study of a codimension three bifurcation which arises in a simple homopolar dynamo model (1) when the coefficient of one of the nonlinear cubic order terms vanishes. In the neighbourhood of the codimension three point we have found exact analytical expressions for the cofficients of the fifth order normal form that describes the behaviour of the system: a normal form which has previously only been studied in abstract by Li and Rousseau [4]. We find, however, that it is possible to relate qualitatively, but not quantitatively, the bifurcation set of the abstract normal form to the

physical problem (compare figure 2 with figure 3). Nevertheless, one aspect of the codimension three analysis is that it provides an explanation of how on 
one side of the codimension three point, i.e. $\epsilon_{3}<0$, the Hopf bifurcation from the trivial state $\left(\mathrm{Hopf}_{1}\right)$ is supercritical, whereas on the other, i.e. $\epsilon_{3}>0$, it is subcritical. Our study shows that, in the full equations, the region of subcriticality is small.

While the unfolding close to the codimension three point predicts a rich structure of behaviour, we have found that for the homopolar dynamo problem this is confined to such a small region of parameter space that it would be hard to observe in practice. There may, however, be other systems for which such a degeneracy would provide more direct comparisons for wider ranges of parameter values. Indeed Moroz [3] showed that a similar degeneracy to the one studied in this paper (as well as the one studied in [2]) could arise in a model for Langmuir circulations. No attempt was made in that paper to analysis the bifurcation structure close to such a degeneracy. The results presented in this

paper, as well as the study of Li and Rousseau [4], should now enable such an analysis to be undertaken.

In a recent study of two homopolar coupled dynamos, Moroz and Hide [7] have shown that the governing (non-degenerate bifurcation) which governs the behaviour is also a Takens-Bogdanov codimension two double-zero bifurcation. Moreover, it possesses precisely the same cubic order degeneracy as the single dynamo system discussed in this paper, and so the bifurcation analysis described above is also applicable.

\section{Acknowledgement}

The authors would like to thank Prof. Raymond Hide, F.R.S. for many interesting discussions. 


\section{References}

[1] R. Hide, A.C. Skeldon and D.J. Acheson, A study of two novel self-exciting single-disk homopolar dynamos: theory. Proc. R. Soc. Lond. A 452, (1996), 1369-1395.

[2] G. Dangelmayr, D. Armbruster and M. Neveling, A codimension three bifurcation for the laser with saturable absorber. Z. Phys. B - Condensed Matter 59, (1985), 365-370.

[3] I.M. Moroz, Codimension three bifurcations arising in Langmuir circulations. Contemporary Mathematics 56, (1986), 223-235.

[4] C. Li and C. Rousseau, Codimension 2 symmetric homoclinic bifurcations and application to 1:2 resonance. Can. J. Math. 42, (1990), 191-212.

[5] J. Guckenheimer and P. Holmes, Nonlinear oscillations, dynamical systems, and bifurcations of vector fields. Applied Math. Sci. 42, (1986), New York: Springer Verlag.

[6] S.-N. Chow, C. Li and D. Wang, Normal forms and bifurcations of planar vector fields. (1994) Cambridge University Press.

[7] I.M. Moroz and R. Hide, On self-exciting coupled double-disk homopolar dynamos driving series motors. Submitted to Physica D. (1997)

\section{Figure Captions}

Figure 1: Qualitative bifurcation set for the normal form of Li and Rousseau $[4]$.

Figure 2: Bifurcation sets in planes of constant $\epsilon_{i}$ computed from the normal form (20) in the case $a_{1}=-1, c_{2}=-1$. (a) $\epsilon_{3}=-0.1$, (b) $\epsilon_{3}=0.1$, (c) $\epsilon_{2}=0.1$.

Figure 3: Bifurcation sets computed from the full equations (equations 1). (a) $\kappa=0.43636, \lambda=0.2$. $\left(\epsilon_{3}=-0.1\right)$. (b) $\kappa=0.07868, \lambda=0.2$. $\left(\epsilon_{3}=0.1\right)$. 